\title{
Collective Shape Oscillations of SiGe Islands on Pit-Patterned Si(001) Substrates: A Coherent-Growth Strategy Enabled by Self-Regulated Intermixing
}

\author{
J. J. Zhang, ${ }^{1, *}$ F. Montalenti, ${ }^{2}$ A. Rastelli, ${ }^{3}$ N. Hrauda, ${ }^{1}$ D. Scopece, ${ }^{3}$ H. Groiss, ${ }^{1}$ J. Stangl, ${ }^{1}$ F. Pezzoli, ${ }^{3}$ \\ F. Schäffler, ${ }^{1}$ O. G. Schmidt, ${ }^{3}$ L. Miglio, ${ }^{2}$ and G. Bauer ${ }^{1}$ \\ ${ }^{1}$ Institut für Halbleiter-und Festkörperphysik, Universität Linz, A-4040 Linz, Austria \\ ${ }^{2}$ L-NESS and Materials Science Department, University of Milano-Bicocca, I-20125 Milano, Italy \\ ${ }^{3}$ Institute for Integrative Nanosciences, IFW Dresden, D-01069 Dresden, Germany \\ (Received 26 May 2010; published 12 October 2010)
}

\begin{abstract}
The shape of coherent $\mathrm{SiGe}$ islands epitaxially grown on pit-patterned $\mathrm{Si}(001)$ substrates displays very uniform collective oscillations with increasing Ge deposition, transforming cyclically between shallower "dome" and steeper "barn" morphologies. Correspondingly, the average Ge content in the alloyed islands also displays an oscillatory behavior, superimposed on a progressive Si enrichment with increasing size. We show that such a growth mode, remarkably different from the flat-substrate case, allows the islands to keep growing in size while avoiding plastic relaxation.
\end{abstract}

DOI: 10.1103/PhysRevLett.105.166102

PACS numbers: 68.55.- - a

Strain-driven self-assembly of three-dimensional Ge or $\mathrm{SiGe}$ islands on $\mathrm{Si}(001)$ substrates is an active topic of both fundamental and practical interest. The $\mathrm{Ge} / \mathrm{Si}(100)$ material system is regarded as prototypical for investigating the basic phenomena leading to the formation and evolution of "self-assembled quantum dots" [1-5]. Technologically, $\mathrm{Ge} / \mathrm{Si}(100)$ is of interest for possible applications in electronic [6,7] and optoelectronic devices [8,9]. As an example, strained Si channels on top of buried, coherent SiGe islands may be used for field effect transistors with enhanced electron mobility [6]. For such an application, sitecontrolled SiGe islands are necessary [10]. Ge growth on Si substrates with regularly arranged pits is a viable path to obtain islands which are not only spatially ordered but also morphologically homogeneous [9-12]. Pits in the Si substrate act as preferential sites for the island formation $[13,14]$ and define equally sized capture zones for the deposited Ge, so that different islands form and grow simultaneously after the completion of a wetting layer. As a consequence, coarsening due to material exchange among islands with dissimilar sizes [3] is largely suppressed [12]. Furthermore, the rate of Ge accumulated within a capture zone can be precisely controlled by changing the Ge flux or the pit period [12]. This is important, since Ge-rich islands with high aspect ratio (height-to-base ratio) are needed as efficient stressors for strained-silicon transistors $[6,15]$.

On both planar and patterned substrates, by increasing the amount of deposited Ge at relatively high growth temperatures, 3D islands evolve from pyramids into domes, and eventually into steeper barns $[4,12]$. This sequence corresponds to a monotonic increase of the aspect ratio, driven by a progressive strain release with island steepness $[3,15]$. With further Ge deposition, the strain energy is eventually released by plastic relaxation $[16,17]$. Plastically relaxed islands display a "cyclic" growth, accompanied by "oscillations" of the island shape after the introduction of each new dislocation [16]. As the growth on pit-patterned substrates has been found to delay the onset of plastic relaxation by lowering the critical elastic budget in the island [18], it would be reasonable to expect aspect ratios beyond the one of barns, in contrast to islands on planar substrates.

Here we show that such a desirable result may be hindered by unexpected shape oscillations during Ge deposition, which nevertheless allow the islands to keep growing in size without plastic relaxation. We observe that with increasing Ge deposition domes transform into barns, then back into domes, and subsequently again into barns, and so on. We determine the 3D composition profiles at different evolution stages by a nanotomography approach based on atomic force microscopy (AFM) [19] and selective wet chemical etching [20], and by x-ray diffraction (XRD) measurements. This analysis clearly shows that the level of intermixing between deposited Ge and Si from the substrate is not constant throughout the deposition in spite of a constant Ge flux and that intermixing leads to Ge distributions in the islands which are more complex than in islands grown on planar substrates. Total energy calculations for different island shapes with realistic volumes show that the shape oscillations can be well explained as due to oscillations of the average $\mathrm{Ge}$ content in SiGe islands with increasing size.

The samples were grown by solid-source molecular beam epitaxy on 2D pit-patterned $\mathrm{Si}(001)$ substrates with periods of 500 and $900 \mathrm{~nm}$. The pit depth and width were about 65 and $350 \mathrm{~nm}$, respectively. Above $45 \mathrm{~nm}$ of $\mathrm{Si}$ buffer, different amounts of Ge [from 9 to 26 monolayer (ML)] were deposited at a substrate temperature of $720^{\circ} \mathrm{C}$ and at a rate of $0.03 \AA / \mathrm{s}$. After growth, the samples were cooled to room temperature for AFM imaging in tapping mode. 
Figures 1(a)-1(f) show AFM images of a sample series obtained after deposition of $9,12,14,17,19$, and $26 \mathrm{ML}$ $\mathrm{Ge}$, respectively, for a pit period of $500 \mathrm{~nm}$. For all investigated Ge coverages from 9 to $26 \mathrm{ML}, 2 \mathrm{D}$ ordered arrays of islands with homogeneous shape are observed. After 9 ML Ge, a uniform array of domes bounded by $\{105\}$, $\{113\}$, and $\{15323\}$ facets is obtained. After $12 \mathrm{ML}$ Ge, steeper $\{111\}$ and $\{20423\}$ facets appear, characteristic for barns. The corresponding surface orientation maps (SOM, see, e.g., Ref. [20]) are plotted in the insets of Figs. 1(a) and 1(b), respectively. Based on previous reports $[4,12,16,17]$, one would expect the transition to even steeper morphologies or dislocation formation with further deposition of Ge. Surprisingly, we find here that after 14 ML Ge barns transform back into domes [see SOM in the inset of Fig. 1(c)]. After $17 \mathrm{ML} \mathrm{Ge,} \mathrm{the} \mathrm{steeper} \mathrm{facets} \mathrm{re-}$ appear again [inset of Fig. 1(d)] and the domes evolve into barns. At 19 ML Ge, barn-shaped islands with additional $\{23420\}$ facets are observed, as illustrated by the SOM in the inset of Fig. 1(e). We denominate this kind of islands as steep barns (SB). By continuing the Ge deposition, the SBs transform back to barns (not shown) which, for $26 \mathrm{ML} \mathrm{Ge}$, transform for a second time back to domes, as shown in Fig. 1(f). It is important to note that such a sequence of transformations occurs without dislocation introduction, as proven by TEM investigations [21].

To distinguish the domes and barns at different stages, we denominate the first appearing domes and barns as $D_{1}$ and $B_{1}$, the second domes and barns as $D_{2}$ and $B_{2}$, and so on. These morphologies are further illustrated by AFM line scans passing through the island centers along the [110] direction [Fig. 2(a)], which clearly show the appearance and disappearance of steep $\{111\}$ facets. When domes transform to barns, the island base width stays almost

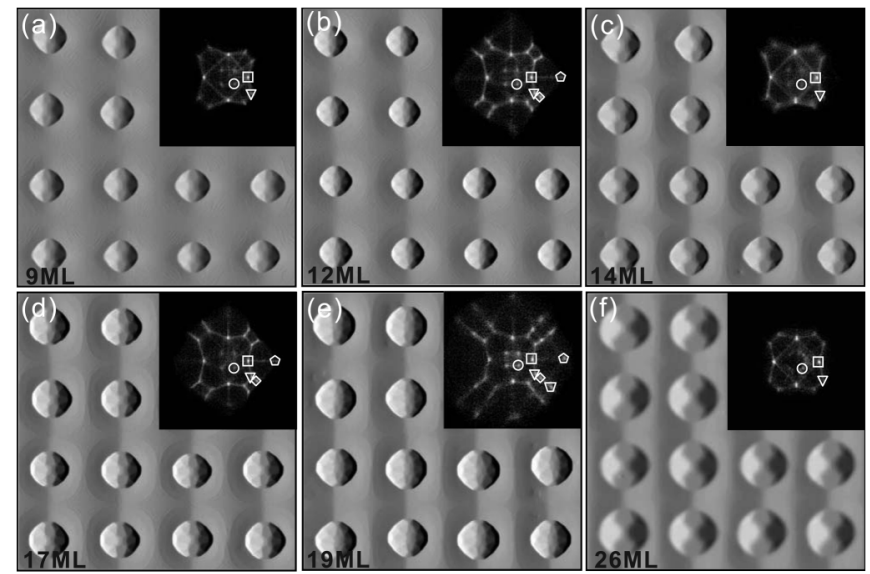

$\mathrm{O}(105) \square(113) \nabla(15323) \diamond(20423) \square(23420) \bullet(111)$

$[110] \longrightarrow-250 \mathrm{~nm}$ Slope $-40^{\circ} \longrightarrow 40^{\circ}$

FIG. 1. AFM images of islands obtained after deposition of 9 (a), 12 (b), 14 (c), 17 (d), 19 (e), and 26 ML Ge (f) on pitpatterned substrates with a period of $500 \mathrm{~nm}$ at $720^{\circ} \mathrm{C}$. The gray scale represents the first derivative along the horizontal axis, and the insets show surface orientation maps with different symbols marking different facets. constant but their height increases. When barns transform back into domes, the island base width increases while the height stays almost the same.

Figure 2(b) displays the island aspect ratio as a function of the Ge amount for growth on patterned substrates with a period of $500 \mathrm{~nm}$ and for a period of $900 \mathrm{~nm}$ under the same growth conditions. For the larger pit period, only one shape oscillation is observed while dislocated islands appear with further Ge deposition, showing that the number of oscillations before plastic relaxation depends on the pattern period. More precisely, at constant Ge supply rate, the rate of $\mathrm{Ge}$ incorporated into islands depends linearly on the capture zone area [12]. Therefore, we expect the Ge incorporation rate for islands on substrates with a pit period of $900 \mathrm{~nm}$ to be by a factor of about 3.2 larger than the corresponding value for a pit period of $500 \mathrm{~nm}$. The larger local Ge flux leads to reduced intermixing, and correspondingly to a higher Ge content [22]. The higher strain associated with the larger Ge content results in an earlier onset of plastic relaxation [23]. The critical role of composition explains why only one shape oscillation is observed for island growth on substrates with the $900 \mathrm{~nm}$ period and possibly also why no shape oscillations were reported for island growth performed at lower temperatures [12].

In order to obtain a more detailed understanding of this effect, we investigated the Ge composition evolution from $D_{1}$ to $D_{3}$ by nanotomography [20]. Figures 3(a)-3(d) show AFM line scans obtained at different steps of etching and the extracted cross-sectional Ge distributions on (110) planes passing through the centers of $D_{1}, B_{1}, D_{2}$, and $B_{2}$ islands, respectively. (For the sake of simplicity, results for
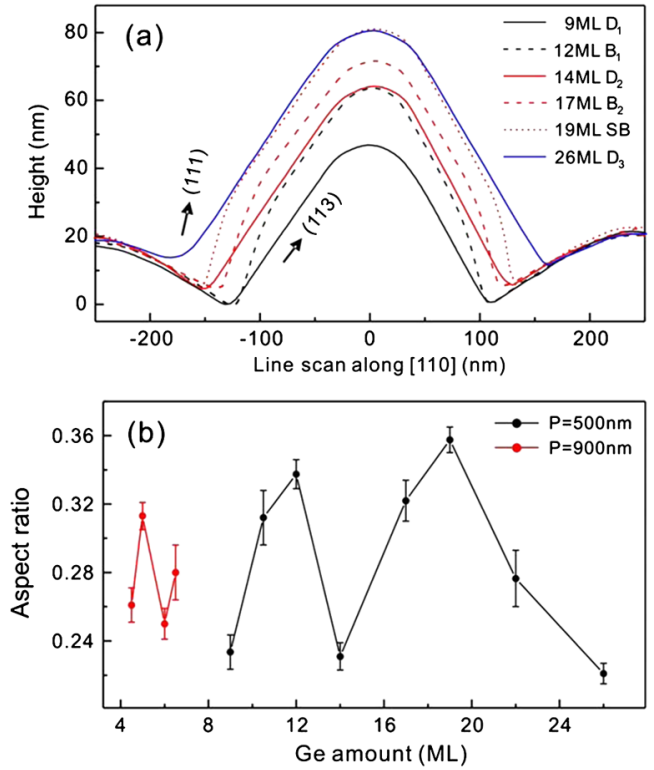

FIG. 2 (color online). (a) AFM line scans passing through island centers along [110] direction for islands shown in Figs. 1(a)-1(f). (b) Island aspect ratio as a function of deposited amount of Ge for pit-patterned substrates with periods 500 and $900 \mathrm{~nm}$, respectively. 
SB and $D_{3}$ are not shown.) The first dome $D_{1}$ shows a composition profile for which the Ge fraction increases rather monotonically along the growth direction, consistent with XRD results for domes grown on patterned substrates [24]. However, the first barn $B_{1}$ shows some deviation from this commonly observed trend: we see in fact a relatively Ge-poor region sandwiched between a Ge-rich top shell and the bottom core [Fig. 3(b)]. This behavior becomes more pronounced for the $D_{2}$ and $B_{2}$ islands. Our XRD measurements [21] further confirm these observations and support the accuracy of the nanotomography results.

As shown in Fig. 3(e), corresponding to the shape oscillations in the sample with pit period of $500 \mathrm{~nm}$, the average Ge content also follows an oscillatory behavior. The average Ge content increases from $D_{1}$ to $B_{1}$, and it drops by more than $4 \%$ when $B_{1}$ transforms back to $D_{2}$ before increasing again when $D_{2}$ evolves to $B_{2}$ and SB. Finally, the evolution of SB into $D_{3}$ is again accompanied by a decrease in Ge fraction by more than $6 \%$. We also note that the average Ge content of $D_{2}$ and $B_{2}$ is lower than that of $D_{1}$ and $B_{1}$, respectively, indicating that the ratio between $\mathrm{Si}$ and $\mathrm{Ge}$ incorporation rates is not constant during the $\mathrm{Ge}$ deposition process. The average incorporation rates of $\mathrm{Si}$ and $\mathrm{Ge}$, calculated from the measured Ge composition distributions, are shown in Fig. 3(f). From the plot we see
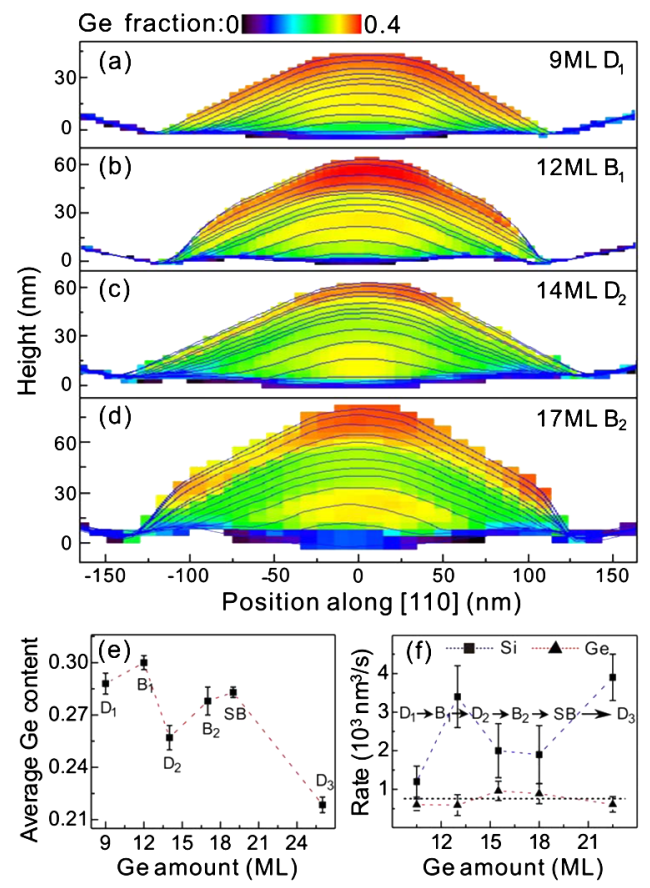

FIG. 3 (color online). (a)-(d) Cross-sectional Ge distributions along [110] direction of the sample with pit period of $500 \mathrm{~nm}$ passing through the island centers for the first dome $D_{1}$ (a), first barn $B_{1}$ (b), second dome $D_{2}$ (c), and second barn $B_{2}$ (d) obtained by selective etching. (e) Average Ge content as a function of deposited $\mathrm{Ge}$ amount for $D_{1}, B_{1}, D_{2}, B_{2}$, steep barn (SB), and third dome $\left(D_{3}\right)$; the dotted line is a guide for the eye. (f) Calculated $\mathrm{Si}$ and $\mathrm{Ge}$ incorporation rates at different stages of Ge deposition; the dashed line shows the nominal Ge incorporation rate. that the $\mathrm{Si}$ incorporation rate in the process leading from $B_{1}$ to $D_{2}$ is $\sim 3$ times higher than the one for the process from $D_{1}$ to $B_{1}$. These values indicate that the $B_{1}$-to- $D_{2}$ shape transformation is associated with a larger Si-Ge intermixing. The same phenomenon is observed for the SB-to- $D_{3}$ transition, and its possible origin is discussed later on. On the other hand, the Ge incorporation rates remain rather constant and close to the expected nominal rate. The latter [see horizontal dashed line in Fig. 3(f)] is estimated from the nominal deposition flux and pattern size, assuming a constant amount of $\mathrm{Ge}$ in the wetting layer.

A total energy calculation based on volumetric strain relaxation and surface energies allows us to estimate how the island shape in a pit depends on volume and average Ge content. Following the experimental data, a barn of composition $x$ is modeled as sitting on an inverted, $\{105\}$ pyramid (fixed composition, $10 \%$ in $\mathrm{Ge}$ ), so that the barn base perimeter is tangential to the square base of the inverted pyramid. If we now transform the barn into a dome of equal $V$ and $x$, but larger in base and smaller in height, the dome base will move upwards and an additional layer of composition $x$ will be added on top of the base of the inverted pyramid (still at 10\% in Ge). Despite the presence of the pit geometry that makes the analysis slightly more complex, the energetic balance between the two islands is analogous to the flat-substrate case $[15,25]$ : steeper islands better relax the elastic-energy density, $\left[\rho_{B}(x)<\rho_{D}(x)\right]$, whereas shallower islands allow for a lower surface energy. If we take the energy difference between the two configurations

$$
\Delta=E_{B}-E_{D}=V\left[\rho_{B}(x)-\rho_{D}(x)\right]+V^{2 / 3} E_{S},
$$

and set it to zero, we obtain for each volume $V_{\text {crit }}$ the average composition $x_{\text {crit }}$ at the border of the stability domains for domes and barns. Here $E_{S}$ includes all the differences in surface terms of the two configurations, including the ones for the pit.

In Fig. 4 we display the morphological stability diagram for barns and domes with a blue dotted line sharing the two domains, as obtained by quantifying the various terms (see supplementary material [21]). In particular, the volumetric elastic-energy terms were evaluated by the finite element method, following the procedure described in Ref. [25]. The surface energies for pit facets are derived from $a b$ initio calculations [26], whereas the average surface energy densities for barns and domes were assumed to be equal $\left(\gamma_{D}=\gamma_{B}=65 \mathrm{meV} / \AA^{2}\right)$ according to Ref. [25]. The maximum error range in our estimations is indicated by dashed black lines in Fig. 4.

By reporting the experimentally measured average concentrations and volumes for $D_{1}, B_{1}, D_{2}, B_{2}, \mathrm{SB}$, and $D_{3}$ in the morphological phase diagram of Fig. 4, we observe that the data points for domes and barns nicely fall into their respective stability regions. We also note that the data point corresponding to the SB with additional steeper facets lies farther away from the blue dotted line compared to $B_{2}$, as 


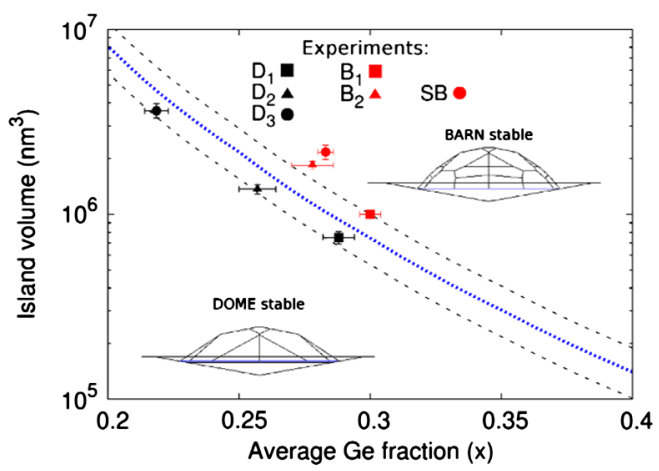

FIG. 4 (color online). Morphological stability diagram for domes and barns, depending on volume and composition, as calculated by the finite element method. The dotted blue line is the domain boundary for $\gamma_{\text {pit }}=62.5 \mathrm{meV} / \AA^{2}$, and the dashed black lines are our maximum estimated error in the surface energies (see text). Experimental values are shown by black ( $D$, domes) and gray (red) ( $B$, barns; $\mathrm{SB}$, steep barns) points, along with their experimental error bars.

expected because of its larger aspect ratio compared to $B_{1}$ and $B_{2}$. Since the experimental data points remain close to the calculated transition line, the balance between Ge flux (provided by deposition) and Si flux (provided by the substrate) determines the morphological evolution: Slight fluctuations in the Ge content with increasing size can trigger the transformation of one shape into the other.

Our interpretation of the collective shape oscillations for coherent islands on patterned substrates is therefore the following. First, the observation of such a phenomenon on pit-patterned substrates is easier with respect to flat substrates because the plastic relaxation is delayed. This is due to (i) the elastic-energy relaxation provided by the shallow pit filled with a Ge-poor $\mathrm{SiGe}$ alloy located below the island (a purely elastic effect) [15], (ii) the larger availability of $\mathrm{Si}$ flow from the faceted pit sidewalls which do not display a homogeneous wetting layer [27], and (iii) the effect of ordered pits in producing islands with very similar properties at all stages of growth. The latter prevents local composition and size fluctuations which are typical for growth on planar surfaces and are responsible for the occurrence of a few plastically relaxed islands even at relatively low $\mathrm{Ge}$ coverages [17].

Second, the small variations in average concentration triggering the morphological transitions are linked to the relative fluxes of $\mathrm{Ge}$ and $\mathrm{Si}$. The latter depends on the growth temperature and varies during Ge deposition. In particular, Fig. 3(f) shows that the Si flux increases considerably whenever islands reach steep morphologies. A possible explanation is that steeper islands, such as barns, are able to carve by compressive load a deeper trench at the base perimeter, expelling $\mathrm{Si}$ out at a higher rate than flatter islands [28]. The expelled Si within a capture zone is then readily available for intermixing with the island material and allows the islands to keep growing in size through shape oscillations. On the contrary, evolution to flatter islands, such as domes, covers the trenches by base expansion, so that the Si flux is drastically reduced.

In conclusion we have reported on the observation of island shape oscillations occurring during the deposition of $\mathrm{Ge}$ on pit-patterned $\mathrm{Si}(001)$ substrates. Such collective oscillations (all islands in the ensemble evolve rather simultaneously) are accompanied by a complex evolution of the Ge distribution and correlate with oscillations in the average $\mathrm{Ge}$ content in the islands. The latter, which are produced by a time-varying $\mathrm{Si}$ flux from the substrate, allow the islands to keep growing in size while avoiding plastic relaxation via efficient $\mathrm{Si}-\mathrm{Ge}$ intermixing. The detailed structural information retrieved from AFM-based nanotomography and $\mathrm{x}$-ray scattering measurements may allow the selection of island growth parameters for best device performance and guide the development of models describing the complex kinetic pathway determining the composition evolution of self-assembled quantum dots.

This work was supported by the FWF, Vienna (SFB025), the EC, Brussels (d-DOTFET), and the DFG (FOR730).

*j.zhang@ifw-dresden.de

[1] Y.-W. Mo et al., Phys. Rev. Lett. 65, 1020 (1990).

[2] G. Medeiros-Ribeiro et al., Science 279, 353 (1998).

[3] F. M. Ross et al., Phys. Rev. Lett. 80, 984 (1998).

[4] A. Rastelli et al., Phys. Status Solidi A 203, 3506 (2006).

[5] M. S. Leite et al., Phys. Rev. Lett. 100, 226101 (2008).

[6] O. G. Schmidt and K. Eberl, IEEE Trans. Electron Devices 48, 1175 (2001); K. W. Ang et al., IEEE Electron Device Lett. 28, 609 (2007); V. Jovanović et al., IEEE Electron Device Lett. 31, 1083 (2010).

[7] G. Katsaros et al., Nature Nanotech. 5, 458 (2010).

[8] V. Lavchiev et al., Opt. Lett. 34, 3785 (2009).

[9] D. Grützmacher et al., Nano Lett. 7, 3150 (2007).

[10] Lateral Alignment of Epitaxial Quantum Dots, edited by O. G. Schmidt (Springer, Berlin, 2007).

[11] Z. Zhong and G. Bauer, Appl. Phys. Lett. 84, 1922 (2004).

[12] J. J. Zhang et al., Appl. Phys. Lett. 91, 173115 (2007).

[13] G. Vastola et al., J. Phys. Condens. Matter 20, 454217 (2008).

[14] H. Hu et al., Phys. Rev. Lett. 101, 216102 (2008).

[15] G. Vastola et al., Self-Assembled Quantum Dots, edited by Zhiming M. Wang (Springer, Berlin, 2008), p. 421.

[16] F. K. LeGoues et al., Phys. Rev. Lett. 73, 300 (1994).

[17] M. Stoffel et al., Phys. Rev. B 74, 155326 (2006).

[18] Z. Zhong et al., Phys. Rev. Lett. 98, 176102 (2007).

[19] R. Magerle, Phys. Rev. Lett. 85, 2749 (2000).

[20] A. Rastelli et al., Nano Lett. 8, 1404 (2008).

[21] See supplementary material at http://link.aps.org/ supplemental/10.1103/PhysRevLett.105.166102 for more details on TEM and XRD measurements and on the model.

[22] E. P. McDaniel et al., Appl. Phys. Lett. 87, 223101 (2005).

[23] A. Marzegalli et al., Phys. Rev. Lett. 99, 235505 (2007).

[24] T. U. Schülli et al., Phys. Rev. Lett. 102, 025502 (2009).

[25] M. Brehm et al., Phys. Rev. B 80, 205321 (2009).

[26] G. H. Lu et al., Phys. Rev. B 72, 125415 (2005).

[27] G. Chen et al., Phys. Rev. B 74, 035302 (2006).

[28] M. Stoffel et al., Phys. Rev. B 75, 113307 (2007). 